\title{
Research on Sanda Athletes' Physical Training Methods
}

\author{
Xiaojun He \\ Weinan Normal University, Weinan, Shaanxi, 714099
}

Keywords: Sanda project; athletes; physical training

\begin{abstract}
Sanda is a combative freehand athletics project that requires the use of certain skills to overcome opponents. Therefore, athletes must not only master certain offensive, counterattack, and defensive skills, but also have a rather high level of physical fitness. With the development of combat training and individualization of modern Sanda project training, athletes must improve their performance to perform reasonable training. The physical fitness of Sanda athletes refers to their physical ability. Without physical support, we will not be able to be talk about skills. The physical shape, physical skills and athletic qualities together constitute the athlete's physical fitness. The three parts are independent and interconnected, and they restrict each other and promote each other. Each part has an impact on the overall level of athletes. This paper focuses on the physical training methods of Sanda athletes.
\end{abstract}

\section{Introduction}

Sanda is a sport that constantly transforms the intensity of exercise. During the competition, opposing players perform freehand exercises based on certain rules, such as kicking, playing, and throwing. In the whole process, the athletes must use their physical fitness, techniques and tactics, exercise intelligence, and strong mentality. The quality of confrontation, and ultimately achieve the purpose of winning opponents. Sanda matches consist of 3 rounds, 3 minutes per round and 1 minute break. During the competition, athletes must master all skills and tactics. They must be highly focused in order to better cope with the various changes on the field; and athletes must have a strong sense of counterattack. On the basis of guaranteeing the strength of punches, it will increase the speed and accuracy of punching. From this it can be seen that during the competition, athletes only have good skills and far from enough. Good physical fitness is also an important factor in winning. Athletes must combine technology and their own overall quality to be more in line with Sanda's characteristics. Therefore, they must Strengthen daily physical training to promote athletes to achieve the perfect combination of speed and strength. In addition, from the analysis of the characteristics of the game, each player in the middle of the game will rest for one minute. At this time, the three energy supply systems of the human body will provide the athletes with the required capabilities in accordance with different proportions. The primary source of phosphoric acid is the main supply. Energy, lactic acid energy system plays a supporting role, and glycolysis energy supply system also plays a key role. It can be seen that physical training of Sanda athletes must also focus on aerobic metabolism and anaerobic metabolism, and according to athletes The actual situation determines the training measures and intensity to improve the athlete's overall competitive ability.

\section{Sanda Athletes' Physical Training Strategies}

Sanda sports training methods are complex in classification and different in pertinence. To facilitate narrative, the following sections mainly discuss the three major modules of physical training: endurance training, speed training, and strength training:

Endurance is a measure of a person's ability to do something or the permanence of a certain action. Endurance quality is the basis of a person's physical fitness, an essential competitive skill for Sanda athletes, and an important physical fitness guarantee for completing Sanda competitions. In the Sanshou project, endurance can be divided into aerobic endurance and anaerobic endurance 
based on the consumption of oxygen by muscle-related exercise. Among them, aerobic endurance has a direct impact on the athletes' implementation of long-lasting technical movements in the competition. That is, the athletes' aerobic endurance is better, and they can show more prominent advantages in a longer period of time. Anaerobic endurance mainly affects the athlete's rapid counterattack ability or defensive ability, such as the player's punching, kicking, distance or frequency of movement. Lung ventilation function, blood oxygenation capacity, cardiac ejection ability, muscle glycogen content, bone metabolism ability and other factors all have a direct impact on aerobic endurance, so we must strengthen the training of heart and lung function and muscle, strengthen the body's Metabolism; anaerobic endurance can also be referred to as weight endurance, that is, the endurance of anaerobic metabolic exercise, long-term supply of energy to muscle contraction under hypoxic conditions requires anaerobic endurance to complete. The endurance training of Sanda athletes can be divided into three parts: the basic phase, the special improvement phase, and the best competition and maintenance phase. The training content and effectiveness of each phase are also different.

Basic endurance training: This stage can use push-ups, continuous semi-squat running, in situ intermittent wheel running, skipping ropes, 800 meters, 3000 meters and other training content, in which push-ups do 3 groups each day, 30 times in each group, can improve athletes Arm endurance; 5 sets of $50-70 \mathrm{~m}$ per day in a row for $50-70 \mathrm{~m}$ each, which can improve the athlete's heart rate control ability; In situ intermittent wheel running 3 sets per day, 50 times per group, can increase leg endurance; skipping rope every day 3 The group, each time 350 times, can train the athlete's speed and endurance; In addition, a group of $800 \mathrm{~m}, 3000 \mathrm{~m}$ runs every day can also train the athlete's circulatory system and lower limb strength. (2) Special improvement phase: Training methods such as push-ups, in-situ intermittent high-legged leg run, in-situ intermittent wheel running, and $100 \mathrm{~m} / 800 \mathrm{~m} / 3000 \mathrm{~m}$ run can be used to perform anaerobic endurance, arm endurance, and lower limb strength exercises. (3) The best competitive and maintenance phase: At this time, it is mainly run at $100 \mathrm{~m} / 800 \mathrm{~m} / 1500 \mathrm{~m}$. It is mainly used for training the combination of endurance and speed. It can also carry out 5000-10000m load-bearing trail running every day, which can effectively improve the athlete's lower limb strength. Train its circulatory system.

For Sanda athletes, they mainly scored by hitting their opponents with arm and leg strength. In the defense process, the arm is an important part of the defense, and the strength index is also an important manifestation of an athlete's basic ability. Therefore, strength training is An important content. In the actual physical training process of the Sanda, the training of strength must be conducted before the training of the large muscle group and then the training of the small muscle group. As long as the large muscle group is fully mobilized, a better exertion effect can be obtained. Therefore, strength training is commonly used. Bench press, flat push, deep squat, and other large muscle groups. The small muscle groups are directly related to the control effect of the technical movements, among which the control of the small muscle groups in the legs is particularly important. According to the stage of the athletes, strength training is also divided into basic training, special training and best competitive and maintenance phases:

Basic strength training: The main training items include push-ups, squats, $50 \mathrm{~m}$ back run, skipping rope, positive pressure leg, side pressure leg, 50m cross step running, vertical jump, frog jump, etc., in which 30 sets of push-ups are done every day 3 groups, mainly training chest, upper arm and other parts; squat training arm, shoulders, legs, back and other parts of the force; 50m return training mainly training leg muscles, skipping main training calf, forefoot; positive pressure leg, The side pressure leg is mainly used to train the ligaments of the thighs. Cross running can improve the coordination of the legs. Vertical jumps and frog jumps can affect the strength of the lower legs and the forefoot. (2) Strength training at special stage: In addition to the training items used in the above basic stage, the training program at this stage will also include jumping steps, bench presses, ping pings, lifting gongs, skipping ropes, and heavy squats. Can affect the leg muscles, bench press main training chest, upper arm strength, flat push can improve the arm, shoulder, leg, back and other parts of the power, lift can increase calf muscle group strength, skipping mainly affects the calf and forefoot, Weight squats affect thigh and psoas muscle strength. 
(3) The best competitive and maintenance phase: mainly use bench presses, flat pushes, lifts, skipping ropes, and weight-bearing squats.

The dodge speed in Sanda is the main factor that determines the quality of attacks, defenses, and movements. Therefore, speed training must be strengthened. Sanda's speed training includes movement speed, reaction speed, and movement speed. The movement speed includes boxing speed, boxing speed, kicking speed, leg taking speed, and technical movement speed. The reaction speed mainly includes the player's own thinking speed. The speed of muscle control and the speed of judgment of the opponent's movement; the speed of movement mainly refers to the attack speed and the speed of movement. Speed training also takes different training measures for different phases.

Basic Training Phase: The basic training items in the basic phase include $50 \mathrm{~m}$ run training, physical orientation training, comprehensive training, lateral or reverse long jump training, frequency conversion training, and chasing training in changing direction. Comprehensive training includes "Zi" running, dodge running, shuttle running, and standing uprights. The main purpose of the above training content is to improve the athlete's movement speed and movement speed, and to exercise their ability to combine speed and endurance. (2) Special improvement phase: The speed training items in the specific improvement phase mainly include bow and arrow rotation, vertical and horizontal jumping body, forward and backward slide, buckling, flying feet, jumping and turning, 50m rapid back run, and Fold back, dodge and touch the shoulders, the above movements mainly train the athlete's movement speed and movement speed, and train 32-4 groups every day. In addition to this, it is also possible to perform assistive combinatorial exercises, such as cross-stepping combination backstepping, sliding-slip cross-stepping, and turning-sliding. At this time, the speed training of the athlete has already had a certain effect, so the training focuses on technology. Mainly, it is necessary to reduce the exercise load. (3) Best competitive maintenance phase: The main training items in this phase include speed ball exercises, 50m sprinting running exercises, boxing gesture exercises, and gassho training. Among them, speedball exercises are performed in groups of 2-5 each day for 1 minute each, 50m sprinting runs. Each day, there are 4 groups, 2 times in each group. Boxers practice 2-4 groups each day, 150-200 times in each group, and practice 2-4 groups with 150-200 times per day. The best competitive maintenance stage is mainly to implement the training results of the basic stage and special improvement stage in the speed of out of the leg and the speed of punching. Through special training, the athletes can quickly respond to the outside world, and can quickly and effectively control their own actions. . During this stage of training, attention should be paid to relaxing muscles and relaxing spirits in order to cultivate calm thinking habits of the athletes. Relaxation of spirits is not ideological slackness, but it is necessary to improve athletes' psychological quality.

\section{Sanda Physical Training Precautions}

The following two issues must be taken into account when performing physical fitness training. On the one hand, physical training should give a scientific evaluation of athletes' body recovery. Physical training can not only pursue high-intensity large-volume exercise. Continuous stimulation may not enable athletes to quickly improve their physical fitness. Recovery and regeneration are also important aspects of physical training. Therefore, it is necessary to make an accurate assessment of the body's recovery of the athletes, to understand the body's reactions during the athlete's physical training through biochemical monitoring, and to understand the body's recovery after the athletes finish training. On the other hand, fully respect the individual differences of athletes. Different athletes have great differences in their body functions. Even if the same athlete is performing the same physical training, there will be some differences before and after training, mainly due to each athlete's way of muscle movement, adaptability, aerobic and anaerobic Metabolism and the ability of the nervous system to control the muscle system are quite different. After the end of training, there will be great differences in the speed of recovery of the athlete's body. Therefore, athletes' physical training should fully respect their individual differences and be formulated for different individuals. Personalized training program. 


\section{Conclusion}

In the Sanda movement, factors such as congenital genetic factors, acquired training effects, number of competitions, training and competition systems, training load characteristics, specialization development trends, nutrition and recovery situations, coaches' personal training philosophy and other factors will all be The physical training effect of athletes has a direct impact. In addition to strengthening regular endurance training, strength training, and speed training in actual training, we must continue to innovate training methods and methods on this basis, scientifically design physical training for free physical exercises, and fully consider the time of competition and energy supply forms of sports events. Factors such as the age factor and skill level of the athlete are used to guarantee the physical training effect of the Sanshou athletes.

\section{References}

[1] Li Huayu. Methods of physical training for young Sanda athletes[J]. Chinese Sports Coach, 2004(4):43- 44.

[2] WANG Xiangquan, SONG Tao, LIU Jian, et al. Study on methods and methods of combination of Sanda athletes' special technology and physical training[J]. Journal of Chengdu Sport University, 2007, 33(6):80- 82.

[3] Xia Junyu. The effect of different training methods on the changes of blood lactate and heart rate among young Sanda athletes[J]. Journal of Nanjing Institute of Physical Education (Natural Science), 2004, 3(4):19- 21.

[4] Jiang Chuanyin, Zhu Xiaoqun, Xie Shouling.Biological research on testing and evaluation of Sanda athletes' training volume[J]. Journal of Shanghai Physical Education Institute, 2004,28(2):2730.

[5] Li Ning. Comprehensive Research on the Scientific Research Tackling and Service of the Elite Sanda Athletes Preparing to Capture Gold in Shaanxi Province[J]. Sports World (Academic Edition), 2011, 11(4):78-79 